\title{
ESTUDIO HISTÓRICO DE LA INTERDISCIPLINARIEDAD DE LAS MATEMÁTICAS BÁSICAS \\ EN LA CARRERA DE ADMINISTRACIÓN DE EMPRESAS DE LA UTEQ EN ECUADOR.
}

\section{HISTORICAL STUDY OF THE INTERDISCIPLINARY RELATIONSHIPS OF THE BASIC MATHEMATICS OF THE CAREER OF BUSINESS ADMINISTRATION IN UTEQ OF THE EQUATOR.}

Edgar Vicente Pastrano Quintana, Mgs. Magíster en Desarrollo Curricular (Ecuador). Magíster en Administración de Empresas (Ecuador). Director de Vinculación de la Universidad Técnica Estatal de Quevedo (UTEQ), Ecuador. epastrano@uteq.edu.ec

José Luis Lissabet Rivero, Dr.C. Doctor en Ciencias Pedagógicas (Cuba). Docente de la Universidad de Granma, Cuba. jlissabet@udg.co.cu

Karina Patricia Arévalo Briones, Mgs. Magíster en Contabilidad y Auditoría (Ecuador). Docente Titular de la Facultad de Ciencias Empresariales de la Universidad Técnica Estatal de Quevedo (UTEQ), Ecuador. karevalo@uteq.edu.ec

\section{ARTÍCULO DE REVISIÓN}

Recibido: 16 de marzo de 2020.

Aceptado: 20 de marzo de 2020.

\section{RESUMEN}

El estudio histórico de interdisciplinariedad en la enseñanza-aprendizaje de Matemáticas Básicas su objetivo es caracterizar históricamente el proceso de establecimiento de relaciones interdisciplinarias de Matemáticas Básicas en Licenciatura en Administración de Empresas de la Universidad Técnica Estatal de Quevedo (UTEQ). Se fundamenta en fuentes documentales con 
normativas metodológicas académicas de la Educación Superior en Ecuador, mediante la utilización de métodos teóricos y empíricos expuestos. Se investigó como período de estudio comprendido desde el año 2000 hasta el 2018, el desarrollo de la carrera de Ingeniería en Gestión Empresarial, vigente con aprobación del proyecto de rediseño, como conclusión se establece que los hitos que marcan inicio de estas etapas son los diferentes modelos de trabajo académico y proyectos de rediseño de la carrera: la primera etapa que corresponde al periodo de los años 2000 al 2011, que incluye la aplicación del Modelo Pedagógico por asignaturas, la segunda etapa desde el año 2012 hasta el 2015 con la aplicación del Modelo Pedagógico por Competencias y finalmente la tercera etapa, desde el 2016 hasta la actualidad, desde la conformación de equipos de trabajos, desarrollo y aprobación del Proyecto de Rediseño Curricular de Licenciatura en Administración de Empresas de la UTEQ.

Palabras clave: estudio histórico, relaciones interdisciplinarias, Matemáticas, enseñanzaaprendizaje, interdisciplinariedad.

\section{ABSTRACT}

The historical study of interdisciplinarity in the teaching learning of Basic Mathematics aims to characterize historically the process of establishing interdisciplinary relations of Basic Mathematics in Bachelor of Business Administration of the State Technical University of Quevedo (UTEQ). It is based on documentary sources with academic methodological regulations of Higher Education in Ecuador, by using exposed theoretical and empirical methods. It was investigated as a study period from 2000 to 2018, the development of the Engineering in Business Management career, in force with approval of the redesign project, as a conclusion it is established that the milestones that mark the beginning of each of these stages are the different models of academic work and career redesign projects: the first stage corresponding to the period from 2000 to 2011 , which includes the application of the Pedagogical Model by subjects, the second stage from 2012 to 2015 with the application of the Pedagogical Model for Competences and finally the third stage from 2016 to the present since the creation of work teams, development and approval of the Curricular Redesign Project of Bachelor of Business Administration of the UTEQ.

Keywords: historical study, interdisciplinary relationships, Mathematics, teaching-learning, interdisciplinarity. 


\section{INTRODUCCIÓN}

Todo proceso o fenómeno educativo tiene su propia historia y, por tanto, puede ser objeto de periodización, pero ésta, para ser verdaderamente científica, debe reflejar las leyes intrínsecas del desarrollo del fenómeno y su relación con el resto del proceso histórico-pedagógico. Enrique Semo afirma que toda periodización es una abstracción por medio de la cual se separa un momento determinado de la historia para otorgarle un carácter de ruptura o viraje.

Por su propia naturaleza, la periodización acentúa la discontinuidad a costa de la continuidad, la estructura en detrimento del proceso, por eso, su validez es relativa. La periodización de la historia, lo que incluye el objeto histórico-educativo, ha planteado siempre grandes problemas, presupone la existencia de un criterio teórico que solo puede derivarse del material histórico, de ahí proviene su relatividad y sus límites.

Para periodizar los antecedentes históricos del objeto de estudio se debe determinar los procedimientos que desarrolla el investigar para realizar una periodización que, con independencia de su reconocida relatividad, pueda hacer argumentada científicamente y aportar el contenido histórico que revele la evolución de su objeto de estudio. Estos procedimientos son: 1.- Precisar el objeto de periodización.

2.- Determinar indicadores de periodización.

3.- Precisión de los hitos históricos o pedagógicos.

4.- Definición rigurosa de los períodos y/o etapas.

En la obra Metodología de la Investigación de la Dra. Aleida Placencia y los Licenciados Oscar Zanetti y Alejandro García se hace referencia a un sistema de conceptos que permiten efectuar las necesarias sub divisiones del proceso histórico y que son: Placencia, A. y otros (1985)
a) La categoría formación económica social.
b) El concepto época histórica.
c) El concepto período.
d) El concepto etapa o fase.

5.- Denominación de los períodos y etapas o fases.

6.- Análisis de los indicadores en cada período y $/ 0$ etapas definidas. Establecimiento de regularidades. 
Como ha planteado el Dr. C. Orlando Valera Alfonso, "en casi ningún texto de Pedagogía se define qué es una tendencia". (Valera, 1998, p. 78) Este autor considera las diferencias que existen entre tendencia pedagógica, referida a la ciencia o ciencias de la educación, a su reflexión sistemática, y tendencia educativa, cuando se refiere a la educación como fenómeno social, a su realización en una sociedad, condiciones, instancias o institución determinada (Valera, 1998).

Entonces, el Dr. Valera define las tendencias educativas como las "posturas teóricas y metodológicas que se asumen ante el fenómeno educativo en un contexto histórico temporal concreto y que se expresan como conceptualizaciones y prácticas educativas determinas. Podemos un poco decir que son modas y modos de educar en un momento determinado. Poseen un mayor grado de generalidad que las pedagógicas, aunque su orientación conceptual es diferente" (Valera, 1998). También plantea que las tendencias pedagógicas "se dan cuando las tendencias educativas se concretan en las corrientes o teorías con el nivel de estructuración propio de la ciencia, lo que da paso a la pedagogía, ciencias pedagógicas o ciencias de la educación. Son, por tato, estructuraciones determinadas de la pedagogía como ciencia, técnica, arte, filosofía y teoría. Tienen menor grado de generalidad que las tendencias educativas y son esencialmente epistemológicas o cienciológicas" (Valera, 1998).

\section{MATERIALES Y MÉTODOS}

En la presente investigación se utilizó el método de análisis histórico-lógico. Lo histórico está relacionado con el estudio de la trayectoria real de los fenómenos y acontecimientos en el decursar de una etapa o período. Lo lógico se ocupa de investigar las leyes generales del funcionamiento y desarrollo del fenómeno, estudia su esencia (Pérez, 1996).

Lo lógico y lo histórico se complementan y vinculan mutuamente. Para poder descubrir las leyes fundamentales de los fenómenos, el método lógico debe basarse en los datos que proporciona el método histórico, de manera que no constituya un simple razonamiento especulativo. De igual modo lo histórico no debe limitarse sólo a la simple descripción de los hechos, sino también debe descubrir la lógica objetiva del desarrollo histórico del objeto de investigación. Si se quiere investigar el desarrollo que ha tenido la educación en un determinado país, en diferentes períodos. Estamos en presencia de una investigación de corte histórico (Pérez, 1996).

Uno de los métodos teóricos fundamentales a utilizar es el método histórico-lógico. El objetivo no es solo describir cómo se ha comportado la interdisciplinariedad de las matemáticas básicas en 
la carrera de Administración de Empresas en la Universidad Técnica Estatal de Quevedo de la República del Ecuador en los períodos estudiados, las condiciones económicas, políticas y sociales que influyeron en los cambios, sino que hace falta también conocer la lógica de su desarrollo, qué elementos de esencia incidieron en los cambios operados en cada etapa.

El estudio de la evolución histórica del proceso del establecimiento de relaciones interdisciplinarias en el proceso de enseñanza-aprendizaje de la asignatura Matemáticas Básicas de la carrera de Licenciatura en Administración de Empresas tiene su fundamento esencial en fuentes documentales relacionadas con normativas metodológicas nacionales del Consejo de Educación Superior (CES) que responden a políticas establecidas de acuerdo con el momento histórico concreto del análisis, además de la utilización de métodos teóricos y empíricos expuestos (Pastrano, 2020).

En este proceso de investigación histórica se tomó como período de estudio el comprendido desde el año 2000 hasta el año 2018, la carrera de Ingeniería en Administración de Empresas, el 2 de Agosto del año 2016 por Resolución 1014_3300 del CES se crea el proyecto de rediseño curricular de la carrera de Licenciatura en Administración de Empresas vigente hasta la actualidad y en el que se manifiesta el desarrollo de la formación del Licenciado en Administración de Empresas en el cantón Quevedo provincia de Los Ríos, República del Ecuador (Dirección Académica-UTEQ, 2016).

Como criterio que precisa la orientación del análisis epistemológico y metodológico a indagar en la valoración histórica se asumió a: el enfoque didáctico-metodológico de tratamiento del contenido la asignatura Matemáticas Básicas de la carrera de Licenciatura en Administración de Empresas, lo que posibilitó precisar los siguientes indicadores:

- Estructuración didáctico-metodológica del proceso de enseñanza-aprendizaje (PEA) de la asignatura Matemáticas Básicas.

- Enfoque seguido en la interrelación de conocimientos de la asignatura Matemáticas Básicas con los conocimientos de las asignaturas de fundamentos teóricos y de la praxis profesional.

- Ejercicios y problemas propuestos en los libros de texto de la asignatura Matemáticas Básicas, y la relación de estos con los contenidos de las asignaturas de fundamentos teóricos y de la praxis profesional de la carrera. 
Las etapas para el estudio histórico se identifican con momentos importantes que denotan la evolución socio-cultural y política de Ecuador, y que inciden en la Educación Superior y en particular en la formación del profesional de la carrera de Licenciatura en Administración de Empresas, las que evidencian cómo se va perfilando hacia el aspecto social debido al contexto del desempeño de este profesional, por lo que los hitos que marcan el inicio de cada una de estas etapas son los diferentes Modelos de trabajo académico y proyectos de rediseño de la carrera.

Primera etapa (2000-2011): Aplicación del Modelo Pedagógico de formación del profesional por asignaturas (MPA) Iniciación de la carrera de Ingeniería en Administración de Empresas (Dirección Académica-UTEQ, 2000).

Segunda etapa (2012-2015): Aplicación del Modelo Pedagógico de formación del profesional por Competencias (MPC) Implementación de los Proyectos integradores en los módulos de la carrera de Ingeniería en Gestión Empresarial (Dirección Académica-UTEQ, 2008).

Tercera etapa (2016-actualidad): Proyecto de Rediseño Curricular Resolución 1014_3300 del CES. Iniciación de la carrera de Licenciatura en Administración de Empresas (Dirección Académica-UTEQ, 2016).

\section{REVISIÓN TEÓRICA}

El estudio de la caracterización histórica del proceso de establecimiento de relaciones interdisciplinarias en el proceso de enseñanza-aprendizaje (PEA) de la asignatura Matemáticas Básicas de la carrera de Licenciatura en Administración de Empresas, en cada una de las etapas e indicadores, se presenta a continuación:

En la primera etapa: iniciación de la carrera de Ingeniería en Administración de Empresas.

En atención al crecimiento de los sectores productivos, sobre la necesidad de contar de sujetos preparados y calificados de acuerdo a las exigencias de una sociedad en plena competencia, el Instituto de Tecnologías de la Universidad Técnica Estatal de Quevedo (UTEQ), incorpora a su currículo de las diferentes Escuelas; carreras terminales en Licenciaturas e Ingenierías (Dirección Académica-UTEQ, 2000).

Con la creación de la Facultad de Ciencias Empresariales, la duración de las carreras terminales se establecieron de ocho y nueve semestres de estudios regulares con una malla curricular de 58 asignaturas, más un año de prácticas pre profesionales, el título a otorgarse fue de Licenciado 
e Ingeniero en Gestión Empresarial, el régimen de estudio fue semestral y las horas de clase tuvieron una duración de 50 minutos cada una, en los horarios de 8:00 a 12:10 con paralelos diurnos y 18:10 a 22:20 en horarios nocturnos en los días de lunes a sábados. Para optar el título de Ingeniero en la carrera ante nombrada, se requería de haber aprobado el pensum académico, aprobar un año de prácticas pre-profesionales, presentar y sustentar un proyecto de graduación (tesis) de conformidad con el reglamento y/o aprobar un seminario de graduación, además aprobar los diferentes niveles de inglés, establecidos para la carrera. El 15 de febrero del 2000 se crea la Facultad de Ciencias Empresariales mediante resolución del consejo universitario (Dirección Académica-UTEQ, 2000).

En esta etapa, este estudio permitió revelar como características a:

Los programas analíticos y sintéticos no profesionalizados, con excesivo volumen de conocimientos, muchos de ellos no esenciales para un Ingeniero en Administración de Empresas en el Ecuador, falta de fundamentación de los contenidos, los conocimientos no constituían un sistema armónicamente integrado y las habilidades no estaban definidas claramente, ni vinculadas con los conocimientos coherentemente, no se precisaban, por tanto, los contenidos generales esenciales y su relación con los conocimientos y habilidades particulares que permitieran su aprobación como medios del aprendizaje, ausencia de problematización, al no declararse explícitamente la necesidad de partir de los problemas profesionales y trabajar en función de su detección, enfrentamiento y solución.

Los programas de las asignaturas carecían de las indicaciones metodológicas necesarias para que el docente pudiera orientarse en la preparación y planificación del proceso de enseñanza aprendizaje, desde un enfoque científico del mismo, el estilo de dirección, los métodos, medios, formas organizativas y el sistema de evaluación los definía cada docente desde su enfoque y criterio personal del proceso, no se realizaba un diagnóstico del desarrollo de los estudiantes, que pudiera considerarse en la planificación del proceso y las necesidades y opiniones de los alumnos no se tuvieron en cuenta en la concepción del proceso de enseñanza aprendizaje , el diagnóstico permitió afirmar que el mismo se caracterizaba por una enseñanza verbalista, reproductiva, memorística y teoricista, en la que el docente ocupaba una posición de poder absoluto y utilizaba un estilo típicamente autoritario, el alumnos era un simple reproductor de información teórica, sometido a las disposiciones, mandatos y decisiones del docente, al que temía intensamente y con el cual no lograba comunicarse adecuadamente, la distancia de la frialdad de las relaciones profesor-alumno era evidente. 
El clima psicológico estaba cargado de tensión, ansiedad y miedo al regaño, a la burla o a la represalia del docente, lo que impedía la espontaneidad, la autenticidad y la participación activa de los alumnos en el proceso mismo del aprendizaje: también había dificultades en la comunicación entre los alumnos.

No se refleja procesos interdisciplinarios establecidos en los programas y planes analítico y sintético de cada asignatura como: fundamentos de administración, contabilidad, metodología de investigación, etc.

Limitados textos para guía en la biblioteca de la Universidad, se ubica libros en los planes en la bibliografía inexistente, los ejercicios y problemas propuestos en los libros de texto de la asignatura Matemáticas Básicas no establecen una relación con los contenidos de las asignaturas de fundamentos teóricos y de la praxis profesional de la carrera.

En la segunda etapa: Implementación de los Proyectos integradores en los módulos de la carrera de Ingeniería en Gestión Empresarial.

En octubre del año 2007, el Honorable Consejo Universitario de la UTEQ aprobó la propuesta para la implementación del MPC y las políticas institucionales para ser aplicadas mediante la asesoría externa de la Dra.C. Gisela Bravo López y MSc. Luis Sánchez Arce. En el documento se establecieron y sistematizaron los contenidos epistemológicos, metodológicos y programáticos de la formación basada en competencias que guardó coherencia con la planificación estratégica y se fundamentó en la necesidad de avanzar en los cambios institucionales iniciados para el aseguramiento de la calidad en la formación profesional de los estudiantes (Dirección AcadémicaUTEQ, 2007).

El Modelo Pedagógico por Competencias (MPC) se basó en el principio de la construcción del conocimiento, ubicó el desarrollo del estudiante en el centro del proceso educativo, para ello, hubo de facilitar que el mismo se descubra como un ser humano en proceso permanente de transformación, de aprendizaje y de crecimiento trascendente a lo largo de la vida, por tanto fue considerado el protagonista de su propio aprendizaje, favoreciendo la toma de conciencia de sí mismo e iniciar así la configuración de un nuevo modelo pedagógico flexible que responda a una concepción nueva de la naturaleza del ser humano y de la sociedad, como realidades en continuo movimiento que interaccionan, se retroalimentan y se sostienen, que permitan la sistematización del MPC, que identifique a la institución en el entorno social y en el país. (Dirección AcadémicaUTEQ, 2007). 
Desde el año 2000, la UTEQ estuvo inmersa en actividades de concreción de los diferentes proyectos propuestos en su Plan de Desarrollo Integral, entre los cuales, se destaca el identificado en el Programa Curricular como: Modelo Pedagógico para la UTEQ.

En septiembre del 2008 se aprueba la implementación del MPC, y el 21 de febrero del 2013 mediante Resolución Segunda se aprueba la revisión curricular 2012-2013 de la carrera de Ingeniería en Gestión Empresarial de la Facultad de Ciencias Empresariales basada en el modelo MPC, según informe de la dirección académica.

Este proceso basado en lo que se ha denominado el Proyecto Tuning. Las cuatro líneas de acción del Proyecto Tuning América Latina son: Competencias (Genéricas y Específicas de las áreas temáticas); Enfoques de Enseñanza, Aprendizaje y Evaluación de estas competencias; Créditos Académicos; Calidad de los programas (Dirección Académica-UTEQ, 2007).

Desde esta perspectiva se hace necesario un cambio en el contexto de la Educación Superior Ecuatoriana el cual exige, formar a los estudiantes en un conjunto de competencias que incluyen conocimientos, habilidades actitudes y valores que los forme para su inserción laboral. Por esta razón, la UTEQ se propuso como objetivo fundamental la elaboración, implementación y evaluación de un MPC que respondiera a las exigencias actuales. El documento que se presenta se ha estructurado en tres capítulos.

En esta etapa este estudio permitió revelar como características a:

Desconocimiento de la aplicación del MPC de parte del docente, falta de capacitación docente sobre didáctica, porcentaje alto de profesionales docentes, docentes resistentes al cambio e innovarse en su práctica docente tradicional, movilidad docente semestre a semestre por renovación de contratos, no se otorgaba unidades de aprendizaje al docente por carrera; por lo general se rellena su carga académica para completar su dedicación, el docente asignado para el desarrollo de las asignaturas no planificaba al inicio sus actividades; en la mayoría de los casos entregaba su planificación después del inicio de sus actividades académicas y algunos eventos no lo hacían laboraban con la de otros docentes, el plan analítico y sintético era el productos de un proceso de copia y pega del documento de anteriores semestres sin actualizarlo, reducido número de textos en la biblioteca de la bibliografía básica y complementaria, el leccionario de trabajo académico no refleja lo que el docente desarrolla en la clase.

Existe incoherencia en la práctica docente sobre la aplicación de métodos, desarrollo de la evaluación no se evalúa resultados de la clase desarrollada conocimientos memorísticos que no 
conducen a desarrollo de competencia generales y específicas, evaluación de aprendizajes no es continua, no refleja el producto del aprendizaje.

El proyecto integrador de cada módulo, siendo el elemento básico para el establecimiento de las relaciones interdisciplinarias, no está fundamentado y concebido en su totalidad por el colectivo de docentes; lo observan como una tarea adicional de fin de semestre, limitada participación de todo el colectivo docente en el proceso de planificación, ejecución, monitoreo y evaluación del proyecto integrador, en la mayoría de los casos solo el director desarrolla estas actividades, no se especifica claramente en cada semestre la competencia específica de cada proyecto integrador, limitados conocimientos sobre el desarrollo de proyectos integradores en el docente asignado, se asigna como relleno de la carga académica los proyectos integradores, se excluye la designación de docentes especialistas de la asignatura profesionalizante del semestre en proyectos integradores, desvirtuándose su accionar durante el semestre, no se actualiza los contenidos del proyecto integrador lo que facilita la repetición de contenidos y el establecimiento de procesos reproductivos por paralelos cerrando la innovación y la creatividad en los estudiantes, la evaluación del docente de los proyectos integradores es anti-técnica e injusta de parte de docente por cuanto durante el proceso existen algunos que no participaron en el proceso de desarrollo y son drásticos en la culminación y entrega del mismo, estudiantes se remiten en el proceso de evaluación del proyecto integrador a preocuparse más de su vestimenta llegando a exhibicionismo extremos, que de la exposición y resultados proyecto.

La mayoría de los docentes coordinadores de los proyectos integradores no ejecutan procesos de revisión de autenticidad y autoría de referencias bibliográficas a través del URKUND, son en un gran porcentaje documentos plagiados, no se fortalece de parte de directivos y sector docente el establecimiento de relaciones interdisciplinarias entre las asignaturas de cada semestre con el proyecto integrador.

Los planes y programas de estudios no reflejan el proceso interdisciplinario entre asignatura en cada semestre.

El docente no presenta en su planificación el establecimiento de objetivos, contenidos, métodos y medios, evaluación y formas organizativas que fundamenten los nodos interdisciplinarios entre las asignaturas.

Los contenidos en los ejercicios y problemas propuestos en los libros de texto de la asignatura Matemáticas Básicas, no tienen la relación con los contenidos de las asignaturas de fundamentos teóricos y de la praxis profesional de la carrera. 
En la tercera etapa: Iniciación de la carrera de Licenciatura en Administración de Empresas.

La UTEQ en base al cumplimiento de las Leyes, Reglamentos y normas legales de la República del Ecuador, emitidas por el Consejo de Educación Superior (CES), Consejo de Evaluación y Aseguramiento de la Calidad de la Educación Superior (CEAACES) y las establecidas en los estatutos y normativas internas, concernientes a aplicar la permanencia y promoción de los docentes procede a través de las unidades académicas a designar equipos de rediseño cuyos referentes se basaron en las políticas de estado de la Revolución Ciudadana a partir de lo establecido y aprobado en la Asamblea de Montecristi: La Constitución de la República del Ecuador, La Ley Orgánica de Educación Superior, Reglamento de Carrera y Escalafón del Profesor e Investigador del Sistema de Educación Superior, Estatuto Orgánico de la UTEQ, Normativa de distribución del tiempo de dedicación de las y los profesores e investigadores de la UTEQ, Reglamento para la Contratación de profesores y profesoras no titulares de la UTEQ.

Con fecha 2 de agosto del 2016 mediante resolución trigésima primera con № 1014-3300 el CES aprueba el Rediseño Curricular de la carrera de Licenciatura en Administración de Empresas con código: 1015-5-650413B01-330.

La carrera de Administración de Empresas con su creación aportará a la solución de diversos problemas que afectan los contextos nacionales, evidenciándose dentro del contexto de economía social, los siguientes: capacidad organizativa, innovación y desarrollo, sostenibilidad y sustentabilidad empresarial, gestión de procesos, comercialización y producción

Los núcleos básicos de la formación del Licenciado en Administración de Empresas establecidos son:

Tres disciplinares propios de la profesión como son: Ciencias aplicadas a las organizaciones, las Tecnologías Informáticas de la Comunicación (TIC) y Epistemología de las ciencias.

Dos básicos que inician en el proceso de formación de los profesionales, relacionados con el objeto de estudio y relacionados con los problemas y necesidades que deben atender los administradores que son: Las ciencias básicas y el estudio social y cultural del hombre.

Los núcleos básicos son los conocimientos cardinales de las disciplinas que permiten la integración de los métodos, lenguajes, procesos y procedimientos que la estructuran. Su producción y aplicación está orientada a la resolución de los problemas de la ciencia y de la realidad relacionados con la profesión. 
El núcleo de las ciencias básicas integra las bases para la comprensión posterior de los conocimientos que abordan los fundamentos de las ciencias, necesarios para comprender los problemas y las necesidades de los contextos disciplinares de la profesión, de modo que resultan propedéuticos para el aprendizaje de los contenidos sustantivos de la carrera, con el fin de sentar las estructuras cognoscitivas para que los estudiantes puedan interactuar eficazmente en ámbitos multidisciplinarios y transdisciplinarios como: Matemáticas (Matemáticas Básicas, Análisis Numérico, Matemáticas Aplicadas a la Administración), Estadística (Estadística Descriptiva, Estadística Inferencial), Economía (Teoría Microeconomía, Teoría Macroeconomía, Política Económica, Economía Popular y Solidaria), Contabilidad (Contabilidad General, Contabilidad Comercial, Contabilidad de Costos.

Las Instituciones de Educación Superior son, en gran medida, entes que contribuyen al cambio de la matriz productiva. El talento humano que se forma en las instituciones debe mostrar las competencias necesarias que les permita ejercer actividades que se vinculen con la matriz productiva. Es por esta razón que esta propuesta de rediseño a la carrera de gestión Empresarial plantea alternativas dirigidas a aportar al cambio productivo de la zona de influencia y productiva de Los Ríos.

El cantón de Quevedo es una zona altamente productiva perteneciente a la Zona 5 del Ecuador. Con este proyecto de rediseño curricular se pretende aportar con propuestas que contribuyan con el desarrollo social, el mejoramiento de la calidad de vida de la población afín al Plan Nacional del Buen vivir (SENPLADES, 2015).

En esta etapa este estudio permitió revelar como características a:

Es inadecuado el método seleccionado para el cumplimiento de objetivos a lograr especialmente en desarrollo de habilidades, inexistencia del sistema de acciones de profesores y alumnos, proceso de enseñanza memorística, bancaria y libresca, las acciones del docente no están dirigidas a estimular y facilitar las acciones de los estudiantes, no se orienta y controla adecuadamente el trabajo independiente, no existe secuencia didáctica de las acciones, en el proceso de enseñanza y aprendizaje del docente no se desarrolla una comunicación con el estudiante y finalmente no se ejemplifica suficientemente y adecuadamente las posiciones teóricas, no se tributa a un PEA desarrollador, las acciones del estudiante no están acorde con las acciones del objetivo y el contenido.

No se concibe la instrumentación y desarrollo de la integración de la asignatura Matemáticas Básicas con otras asignaturas en la carrera, en las actividades docentes y extradocentes, no se 
realiza la determinación y formulación del objetivo de la clase, en la asignatura Matemáticas Básicas, a partir del establecimiento de relaciones interdisciplinarias, el docente no estructura el contenido de la enseñanza en la clase, a partir del establecimiento de relaciones interdisciplinarias.

El docente concibe un limitado sistema de métodos, medios y procedimientos de enseñanza y aprendizaje que utiliza en la clase, a partir del establecimiento de relaciones interdisciplinarias, inexistencia de formas de organización de la enseñanza en la clase de Matemáticas Básicas, en función del logro de las relaciones interdisciplinarias, limitada aplicación del sistema de evaluación de aprendizaje mediante el empleo de técnicas evaluativas a partir de relaciones interdisciplinarias.

Limitados elementos o aspectos de carácter didáctico-metodológico que debe tomar el docente en consideración para lograr en los estudiantes su motivación e implicación, en la realización de la tarea docente, la construcción y apropiación de los conocimientos, la sistematización del contenido, la aplicación de los conocimientos a la solución y valoración de situaciones de la práctica social en la clase a partir del establecimiento de relaciones interdisciplinarias y finalmente el desarrollo y la consolidación de cualidades de la personalidad de los estudiantes en las esferas cognitivo-intelectual, afectivo-volitivas y socio-moral, en la carrera de Administración de Empresas.

Los ejercicios y problemas propuestos en la bibliografía básica y complementaria de los sílabos de la asignatura Matemáticas Básicas, no se establecen una relación interdisciplinaria con los contenidos de las asignaturas de fundamentos teóricos y de la praxis profesional de la carrera de administración de empresas de la universidad.

Este estudio de la caracterización histórica realizado es uno de los elementos fundamentales del proceso de diagnóstico del establecimiento de relaciones interdisciplinarias en la asignatura Matemáticas Básicas, de la carrera de Licenciatura en Administración de Empresas, lo que ha permitido comprenderlo en su historia, en su desarrollo y en su lógica y, por tanto, determinar, fundamentar y formular el problema de la investigación enunciado en la introducción. 


\section{CONCLUSIONES}

Se puede concluir que los hitos que marcan el inicio de cada una de estas etapas son los diferentes Modelos de trabajo académico y proyectos de rediseño de la carrera que son:

La primera etapa que corresponde al periodo de los años 2000-2011, que incluye la aplicación del MPA y la iniciación de la carrera de Ingeniería en Administración de empresas, como ingeniería en gestión empresarial

La segunda etapa desde el año 2012 hasta el 2015 con la aplicación del MPC e implementación de los Proyectos integradores en los módulos de la carrera de Ingeniería en Gestión Empresarial La tercera etapa desde el 2016 hasta la actualidad mediante la conformación de equipos de trabajos en el interior de la facultad y el debido proceso de aprobación del Proyecto de Rediseño Curricular con Resolución 1014_3300 del CES e Iniciación de la carrera de Licenciatura en Administración de Empresas

\section{REFERENCIAS BIBLIOGRÁFICAS}

Dirección Estratégica-UTEQ. (2000). Plan Desarrollo Integral de la UTEQ. Quevedo, Ecuador: Editorial Universitaria UTEQ.

Dirección Académica-UTEQ. (2000). Proyecto de Creación de Facultad de Ciencias Empresariales. Quevedo - Ecuador: Editorial Universitaria UTEQ.

Dirección Académica-UTEQ. (2007). Proyecto de Creación e implementacion del Modelo MPC de la UTEQ. Ecuador: Editorial Universitaria UTEQ

Dirección Académica-UTEQ. (2013). Revisión del Proyecto de Creación de la Carrera de Ingeniería en Gestión Empresarial de la Facultad de Ciencias Empresariales. Ecuador: Editorial Universitaria UTEQ

Dirección Académica-UTEQ. (2016). Proyecto de Rediseño curricular de la carrera de Licenciatura en Administración de Empresas. Ecuador: Editorial Universitaria UTEQ.

Gastón, G. (1996). Metodología de la investigación educacional. La Habana (Cuba), ECURED. 
Pastrano Quintana, E. (2020). Modelo didáctico de tratamiento interdisciplinar del contenido de la asignatura matemáticas básicas de la carrera de licenciatura en administración de empresas, UTEQ, República de Ecuador. Tesis en opción al grado científico de Doctor en Ciencias Pedagógicas. Universidad de Granma, Cuba.

Placencia, A. y otros (1985). Metodología de la investigación histórica. La Habana(Cuba): Editorial Pueblo y Educación.

Placencia, A. (1975). Método y Metódica en Historia. Cuba, La Habana: Editorial Pueblo y Educación.

Ramos, G. (2002). Aproximación a una metodología para el análisis de los antecedentes históricos en la investigación pedagógica. Cuba: Editorial NN.

SENPLADES. (2015). Plan Nacional del Buen Vivir. Vicepresidencia de la República del Ecuador. Ecuador: Registro Oficial del Ecuador.

Valera, O. (1995). Estudio critico de las principales corrientes de la psicología contemporánea, México: Editorial Pueblo y Educación.

Valera, O. (1998). Las tendencias pedagógicas contemporáneas. La Habana (Cuba): Editorial Pueblo y Educación. 